\title{
Desenho Universal para Aprendizagem, Acessibilidade Web, Usabilidade no e- Learning e Usabilidade Pedagógica
}

\section{Universal Design for Learning, Web Accessibility, Usability in e-Learning and Pedagogical Usability}

\author{
Viviane Silva ${ }^{*, * *}$, Maria Gomes*, Ranniéry Souza*;*** \\ *Universidade do Minho, **Instituto Federal de Educação do Amazonas, ***Universidade do Estado do Amazonas
}

\begin{abstract}
Resumo
Este artigo analisou os conceitos - "Desenho Universal para Aprendizagem"; "Acessibilidade na Web"; "Usabilidade no e-learning"; e "Usabilidade Pedagógica" - com o objetivo de identificar a articulação destes conceitos no contexto do Ensino a Distância (EaD) notadamente no e-learning destinadas ao aperfeiçoamento de Ambientes Virtuais de Aprendizagem. Para lograr este intento utilizou-se a metodologia de revisão sistemática da literatura fundamentada em Tranfield, et. al (2002). A pesquisa usou como lócus as bases científicas: Scopus, Web of Science e Google Scholar, no intervalo de 2012 a 2017. Nas pesquisas identificaram-se inicialmente 96 artigos em que foram selecionados, após uma análise preliminar, 14 artigos cujo conteúdo constituiu o corpus de suporte a este texto. Para futuros estudos sugere-se alargar o escopo da pesquisa.

Palavras clave: Design Universal para Aprendizagem, Acessibilidade na Web, Usabilidade no e-learning, Usabilidade Pedagógica.
\end{abstract}

\begin{abstract}
This article analyzed the concepts - "Universal Design for Learning"; "Web accessibility"; "Usability in e-learning"; and "Pedagogical Usability" - with the objective of identifying the articulation of these concepts in the context of Distance Learning (EaD), especially in elearning aimed at improving Virtual Learning Environments. In order to achieve this, the methodology of systematic review of the literature based on Tranfield, et. al (2002). The research used as a locus the scientific bases: Scopus, Web of Science and Google Scholar, in the interval of 2012 to 2017 . In the researches, 96 articles were initially identified in which, after a preliminary analysis, 14 articles were selected whose content constituted the corpus support this text. For future studies it is suggested to widen the scope of the research.

Keywords: Universal Design for Learning, Web Accessibility, Usability for e-learning, Pedagogical Usability
\end{abstract}

\section{Introdução}

A criação da interface Web na década de 90, pelo físico Tim Berners-Lee e sua equipe no CERN European Organization for Nuclear Research, foi concebida e idealizada como: "The power of the Web is in its universality. Access by everyone regardless of disability is an essential aspect" (W3C, 1997). Contudo, o objetivo idealizado para a Web ainda não foi alcançado de maneira efetiva e igualitária, especialmente no contexto educacional, apesar dos esforços empreendidos nas últimas décadas. Entretanto há crescente procura pelos contextos de Educação a Distância (EaD), notadamente na modalidade de elearning, a qual, segundo Gomes (2005), está intrinsecamente associada à Internet e ao serviço WWW. Este crescimento ocorre em múltiplos países como evidenciam os dados estatísticos de diversos estudos (conf. Linder, Fontaine-Rainen, \& Behling 2015; NCES, 2014; Open University, 2015; SEMESP, 2016).

Apesar do expressivo aumento na procura pelo elearning, da qual uma das causas é a expansão da Internet e dos serviços e funcionalidades que lhe estão associadas e às quais estão agregadas diversas vantagens como sejam a flexibilidade de local para estudo, autonomia, gerenciamento do tempo e economia de recursos para locomoção, ainda são, qualitativa e quantitativamente, insuficientes as pesquisas científicas destinadas ao aperfeiçoamento de Ambientes Virtuais de Aprendizagem (AVA), em especial aquelas com foco na procura da satisfação do estudante e na diminuição dos índices de abandono e desistência, bem como os estudos com foco em políticas inclusivas, nomeadamente de estudantes que possuam alguma deficiência seja física, de aprendizagem, cognitiva, mental, social, afetiva ou cultural.

$\mathrm{Na}$ base do desenvolvimento de vivências de aprendizagem em modalidade de e-learning que se revelem mais satisfatórias e mais inclusivas, consideramos fundamental ter presente um conjunto de princípios associados aos conceitos de Desenho Universal para Aprendizagem, Acessibilidade na Web, Usabilidade no e-learning e Usabilidade Pedagógica. Nesse sentido, consideramos que se torna relevante clarificar estes conceitos e identificar a sua presença nas publicações acadêmicas com foco no e-learning. Com esse desiderato, levamos a cabo um estudo de revisão de 
sistemática da literatura que nos revelasse como as Instituições de Ensino (IE) estão a considerar as implicações desses conceitos nas configurações das suas práticas de e-learning com base em AVA.

Para além desta introdução, este artigo descreve a metodologia do estudo; expõe sucintamente os conceitos em consideração, apresenta e analisa os principais dados coletados e conclui com algumas reflexões finais.

\section{Metodologia do estudo}

A metodologia do estudo consistiu numa Revisão Sistemática da Literatura (RSL) junto às bases de dados Web of Science, Scopus e Google Scholar, adotando um protocolo de pesquisa replicável, baseado em uma adaptação de métodos de RSL de aplicação nas áreas de Ciências Sociais (Tranfield et al., 2002). Esta RSL foi delimitada ao período de 2012 a 2017 e traçou um panorama de artigos oriundos de autores em diferentes países e continentes, os quais se reportassem a estudos cujo foco fosse a aplicação dos conceitos subjacentes às expressões de pesquisas utilizadas, a casos e contextos específicos de $\mathrm{EaD}$ em modalidade de e-learning com suporte em AVA.

As expressões de pesquisa adotadas na RSL foram as seguintes: Universal Design for Learning, Web Accessibility for learning, Usability for e-learning, Pedagogical Usability. Como critérios de inclusão foram considerados os artigos referenciados no domínio da Tecnologia Educativa, e Educação e Tecnologia; em idioma Português ou Inglês; publicados entre 2012 e 2017 e que estivessem com acesso aberto nas bases de dados pesquisadas. Foram excluídos artigos fora dos domínios atrás referidos (por exemplos artigos com ênfase na análise de hardware ou infraestruturas físicas); artigos duplicados; artigos com menos de 10 citações no Google Scholar e artigos com acesso restrito.

O resultado inicial apontou para 96 artigos, dos quais, após a leitura de cada resumo, se selecionaram os artigos que efetivamente mobilizavam os conceitos em causa, em contextos de e-learning num total de 14 trabalhos que se consideraram com alta relevância, quer pelo impacto e número de citações, quer pela diversidade de perspectivas, para uma análise mais aprofundada. Este conjunto de 14 textos constituíram o corpus de análise deste artigo.

\section{Desenho Universal para Aprendizagem}

O conceito de Desenho Universal surgiu na década de 60, desenvolvido por profissionais da área de arquitetura na Universidade da Carolina do Norte - EUA, com objetivo de definir projetos de produtos e ambientes para serem usados por todos, na sua máxima extensão possível, sem necessidade de adaptação ou projeto especializado para pessoas com deficiência (Carletto \& Cambiaghi, 2008).

Desde a Conferência Mundial sobre Educação para Todos (UNESCO,1990) realizada com a preocupação fundamental de universalizar o acesso à educação para todos, assegurando que todas as pessoas tenham oportunidades educativas que vão ao encontro das suas necessidades específicas de aprendizagem e promovendo a equidade em termos educacionais, que o conceito de educação inclusiva angariou particular importância na agenda de organismos internacionais. Esse fato contribuiu para que o termo Desenho Universal migrasse para o contexto educativo, sob a designação de Desenho Universal para a Aprendizagem (DUA).

O Center for Applied Special Technology (CAST) define Desenho Universal para Aprendizagem (DUA), como: "Universal design for learning is a framework to improve and optimize teaching and learning for all people based on scientific insights into how humans learn" (CAST, 2013).

O Desenho Universal para Aprendizagem implica o desenho de ambientes e experiências de aprendizagem que sejam capazes de envolver e motivar todos os aprendizes, independentemente da diversidade de características e preferências dos mesmos, o que torna essencial considerar a existência dessas diferenças de modo a que as experiências de aprendizagem sejam ajustadas às diferentes necessidades individuais (CAST, 2011)

\section{Acessibilidade na Web}

O termo "acessibilidade" surgiu no final da década de 40, para designar a condição de acesso a locais, produtos, serviços ou informações, ao maior número e variedade possível de pessoas, independentemente de suas capacidades físico-motoras e perceptivas, culturais e sociais. Sendo a Web usada por um número cada vez maior de pessoas de diferentes idades, status social, culturas, etnias e características físicas e cognitivas (Katsanos, Tselios, Tsakoumis, \& Avouris, 2012). O grupo oficial que desenvolve as recomendações de acessibilidade na Web é o Grupo de Trabalho das Diretrizes de Acessibilidade para o Conteúdo da Web (WCAG, 2008) integrante do World Wide Web Consortium (W3C) (W3C, 2016).

\section{Usabilidade técnica e usabilidade pedagógica}

O termo usabilidade surgiu na área de Interação Humano Computador (IHC) na década de 80 e seu uso foi ampliado com a criação de interfaces gráficas que facilitam aos aprendizes a interação em Tecnologias de Informação e Comunicação (TIC).

A norma ISO 9241 (1998) define a usabilidade como "a medida em que um produto pode ser usado por usuários especificados para atingir metas especificadas com eficácia, eficiência e satisfação em um contexto de uso específico". A usabilidade de uma interface que envolve não apenas medir questões relativas às funcionalidades do software, mas também a facilidade de seu uso, e tem como principal desafio reduzir o tempo necessário de aprendizagem para utilização do sistema (Nielsen, 1993).

Silius \& Tervakari (2003) afirmam que é importante avaliar o desenho pedagógico de sistemas e-learning, para além da usabilidade técnica, que é um requisito indispensável para qualquer software. O termo Usabilidade Pedagógica (UP) indica se as ferramentas, o conteúdo, a interface e as tarefas nos ambientes de 
aprendizagem, baseados na Web, apoiam estudantes na aprendizagem em vários contextos, de acordo com objetivos pedagógicos selecionados e destacam também que uma plataforma de aprendizagem pode ser utilizável, mas não ser pedagogicamente benéfica e vice-versa, embora possa haver alguma sobreposição nos problemas identificados (Silius \& Tervakari, 2003). A avaliação da UP das aplicações de e-learning merece atenção especial, e os avaliadores precisam de metodologias efetivas e diretrizes adequadas para realizar tal tarefa (Çelik, 2012).

O conceito de UP contribui diretamente para o design do AVA, do material didático digital e das atividades pedagógicas e adequadas aos cenários e contextos em elearning, além de tornar o uso do ambiente gratificante $\mathrm{e}$ estimulante para os participantes envolvidos nos mesmos com o propósito de alcançar os objetivos e metas de aprendizagem (Silva, Hilário, Souza, \& Gomes, 2017).

\section{Discussão dos resultados}

Destacamos como um dos resultados desta RSL, que o conceito "acessibilidade na Web" foi o mais discutido entre os autores dos trabalhos selecionados, surgindo em $36 \%$ dos artigos encontrados (conf. Fig. 1), e revelando a preocupação das IE em atender a normas legais e/ou adequar o ambiente e-learning para inclusão de alunos com deficiência.

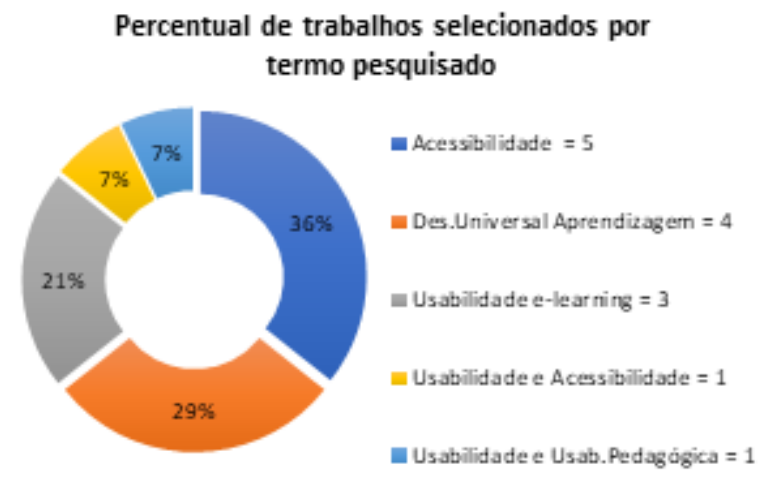

Figura 1. Gráfico com percentual de trabalhos selecionados por termo pesquisado

O conceito de "Desenho Universal de Aprendizagem", é central em $29 \%$ dos artigos, com foco na atenção em implantar o conceito de DUA no elearning e desse modo tornar este cenário educativo mais inclusivo. O termo Usabilidade associado ao elearning e com a aplicação na perspectiva pedagógica no AVA, surge em $21 \%$ dos trabalhos selecionados. Uma única publicação apresenta os conceitos Usabilidade articulado a Acessibilidade e Usabilidade vinculado a Usabilidade Pedagógica, representando simultaneamente $7 \%$ dos trabalhos selecionados.

Na perspetiva do conceito "Acessibilidade na Web" - das cinco publicações, destacamos os principais achados. a.) A maior parte dos artigos foram direcionados à temática Acessibilidade Web para adequar o AVA, tanto em plataformas de acesso livre quanto em ambientes proprietários (restritos), principalmente para torná-los inclusivos, agradáveis e motivadores, bem como para atender a legislação vigente do local e ampliar a oferta de e-learning em instituições de ensino superior (IES).

b.) A OU, desde a implantação da Lei da Igualdade em 2010 (OU, 2010) nas IES no Reino Unido, que passou a exigir das universidades condições que evitem a discriminação e que forneçam condições de maior e melhor acesso à educação, tem priorizado investimentos em tecnologias com acessibilidade e infraestrutura adequada (Slater, Pearson, Warren, \& Forbes, 2015).

c) $\mathrm{Na}$ perspectiva da adoção das diretrizes de acessibilidade convencionadas internacionalmente, o estudo realizado por Linder et al. (2015) indica que inúmeras IES nos EUA carecem de infraestrutura básica para ampliar as condições de acessibilidade no elearning. Segundo Linder et al., (2015) em seu estudo foram encontrados três desafios: (1) necessidade de identificar o responsável pela implementação de políticas de acessibilidade na Web; (2) necessidade de investimento institucional ao criar ambientes online que sejam acessíveis em termos de tecnologia, pessoal e outros recursos; e (3) necessidade de esclarecimento dos responsáveis para iniciativas de aprimoramento dos itens de acessibilidade, esta situação gera não raro certo grau de intolerância da parte destes profissionais para estas iniciativas de melhoramento.

Na perspectiva do conceito "Desenho Universal para Aprendizagem" - das quatro publicações, destacamos os principais achados de dois autores selecionados:

a) O trabalho de Reid et al. (2013) discute o papel da tecnologia móvel em apoiar pessoas com dislexia dentro do quadro teórico do DUA. Os autores discutem como os alunos com dislexia podem usar a tecnologia móvel para abordar uma ampla gama de necessidades acadêmicas (como ler, compor texto, tomar nota, metacognição e competências de estudo). O artigo descreve 10 aplicativos de tecnologia móvel para apoiar a aprendizagem por parte de alunos com dislexia, dentro e fora da sala de aula, e relata a importância do professor conhecer as aplicações em tecnologias móveis e a parceria que deve haver entre a escola, professores, coordenadores e pais para alcançar os resultados desejados da aprendizagem.

b) O estudo de Erkilic \& Durak, 2013, abordou o Desenho Universal sob a perspectiva arquitetônica da IE e problemas espaciais de inclusão em seus ambientes educacionais (Erkilic \& Durak, 2013) e constatou que há falta de clareza na compreensão sobre educação inclusiva e como atender aos conceitos do DUA.

Na perspectiva do conceito de "Usabilidade no elearning" - das três publicações, destacamos os principais achados de dois autores. 
a) Segundo Green, Inan, \& Denton (2012) a satisfação dos alunos pode ser claramente vinculada à usabilidade, disponibilidade e qualidade da assistência técnica e frequência de uso. Neste estudo é enfatizado que o sistema seja utilizável, que os alunos tenham acesso ao suporte técnico e que sejam encorajados a usar o sistema com frequência, desta forma poderá ocorrer uma aprendizagem online de qualidade.

b) No trabalho de Orfanou, Tselios, \& Katsanos (2015) de avaliação de usabilidade foi aplicado o método SUS - System Usability Scale - que é um dos mais conhecidos e simples métodos de averiguação do nível de usabilidade de um sistema. É possível aplicar o método SUS, para avaliar produtos, serviços, hardware, software, websites, aplicações - e qualquer outro tipo de interface, porém para sistemas de e-learning o SUS não apresenta todos os recursos necessários para a aprendizagem.

Articulando as perspectivas dos conceitos de "Acessibilidade e Usabilidade" - uma publicação foi encontrada. No estudo de Hollins \& Foley (2013) foi percebido que pode ser útil para as IES estabelecer políticas que abordem o uso de diretrizes de usabilidade e acessibilidade articuladas no projeto do campus virtual.

Articulando as perspectivas de "Usabilidade e Usabilidade Pedagógica", identificou-se a publicação de Çelik (2012) destacando que as plataformas elearning devem ser desenvolvidas para serem centrados no aluno, serem pertinentes, motivacionais e capazes de acomodar rotinas individuais de estudo de alunos, estando preparadas para serem o mais flexíveis possível. A avaliação de software educacional para e-learning deve considerar sua usabilidade e sua eficácia pedagógica, ou seja sua usabilidade pedagógica (Çelik, 2012).

\section{Considerações finais}

No nosso entendimento, todos os conceitos considerados neste estudo e que deram origem às expressões de pesquisa na base da RSL realizada são conceitos relativos ao aprimoramento da qualidade no elearning. A análise aos textos selecionado aponta também nesse sentido tendo-se identificado uma ideia comum na direção da necessidade de aperfeiçoamento dos ambientes e práticas de e-learning de modo a tornálo mais inclusivo, motivador e agradável aos usuários, no propósito de gerar melhor condições de aprendizagem. Talvez por isso, e também impelidos ao cumprimento das diretrizes internacionais convencionadas por organismos como UNESCO e W3C, o maior número de trabalhos estão voltados para a Acessibilidade na Web e Desenho Universal para Aprendizagem.

Embora consideramos que todos os conceitos em causa estão estritamente relacionados e devem articularse no sentido de um desenvolvimento e avaliação de experiências de aprendizagem em modalidade de elearning numa perspetiva holística, nenhum dos textos analisados se reportou a estudos com todos os conceitos articulados em um único projeto, patenteando assim uma visão científica divergente à visão sistêmica.

Por fim, consideramos digno de nota a desatenção por parte das comunidades científicas aqui analisadas, referente aos conceitos de Usabilidade no e-learning e Usabilidade Pedagógica, posto que como já referimos um único trabalho pesquisou estas temáticas. Parece-nos que, ou a compreensão conceitual de Usabilidade Pedagógica ainda está a ser parcial, ou, está a ser confundida com outros conceitos.

Acreditamos que todos os conceitos pesquisados contribuem para o aprimoramento do e-learning, seja para alunos com ou sem deficiência, permitindo alternativas de representação do conhecimento, do conteúdo, da avaliação em formatos diversos e agregando a facilidade de uso, facilidade de aprendizado e principalmente satisfação no acessar o e-learning. Como trabalhos futuros evidenciamos a necessidade de alargar o escopo da pesquisa por um maior período e incluir outras bases de dados científicas. Como nota final, registra-se o limite de dimensão imposto ao texto que obrigou a uma apresentação sucinta dos dados recolhidos.

\section{Referencias}

Carletto, A. C., \& Cambiaghi, S. (2008). Desenho Universal: Um conceito para todos. São Paulo: Instituto Mara Gabrilli. Recuperado de http://www.vereadoramaragabrilli.com.br/files/univer sal_web.pdf

CAST. (2011). National Center on Universal Design for Learning. Recuperado 3 de setembro de 2017, de http://www.udlcenter.org/aboutudl/udlguidelines/prin ciple3

CAST. (2013). About Universal Design for Learning. Recuperado 27 de junho de 2017, de http://www.udlcenter.org/aboutudl/whatisudl

Çelik, S. (2012). Development of usability criteria for elearning content development software. Turkish Online Journal of Distance Education, 13(2), 336345. Recuperado de dergipark.ulakbim.gov.tr/tojde/article/view/50001023 27

Erkilic, M., \& Durak, S. (2013). Tolerable and inclusive learning spaces: an evaluation of policies and specifications for physical environments that promote inclusion in Turkish Primary Schools. International Journal of Inclusive Education, 17(5), 462-479. http://doi.org/10.1080/13603116.2012.685333

Gomes, M. J. (2005). E-Learning: Reflexões em Torno do Conceito. Paulo Dias e Varela de Freitas (orgs.). Atas da IV Conferência Internacional de Tecnologias de Informação e Comunicação na Educação Challenges'05, 229-236. http://doi.org/10.1017/CBO9781107415324.004

Green, L. S., Inan, F. A., \& Denton, B. (2012). Examination of factors impacting student satisfaction with a new learning management system. Turkish Online Journal of Distance Education, 13(3), 189197 Recuperado de dergipark.ulakbim.gov.tr/tojde/article/view/50001023 
$46 / 0$

Hollins, N., \& Foley, A. R. (2013). The experiences of students with learning disabilities in a higher education virtual campus. Educational Technology Research and Development, 61(4), 607-624. http://doi.org/10.1007/s11423-013-9302-9

Katsanos, C., Tselios, N., Tsakoumis, A., \& Avouris, N. (2012). Learning about web accessibility: A project based tool-mediated approach. Education and Information Technologies, 17(1), 79-94. http://doi.org/10.1007/s10639-010-9145-5

Linder, K. E., Fontaine-Rainen, D. L., \& Behling, K. (2015). Whose job is it? Key challenges and future directions for online accessibility in US Institutions of Higher Education. Open Learning: The Journal of Open, Distance and e-Learning, 30(1), 21-34.

NCES. (2014). U.S. Department of Education's National Center for Educational Statistics. Recuperado 29 de agosto de 2017, de https://nces.ed.gov

Nielsen, J. (1993). Usability engineering. Boston: Academic Press. Recuperado de http://dl.acm.org/citation.cfm?id=529793

Open University. (2015). Open University United Kingdom. Recuperado 17 de dezembro de 2015, de http://www.open.ac.uk/about/main/strategy/facts-andfigures

Orfanou, K., Tselios, N., \& Katsanos, C. (2015). Perceived Usability Evaluation of Learning Management Systems: Empirical Evaluation of the System Usability Scale. International Review of Research in Open and Distributed Learning, 16(2), 227-246.

OU. (2010). Equality Act. Recuperado 27 de junho de 2017, de http://www.open.ac.uk/equalitydiversity/content/equality-law-1

SEMESP, S. das M. de E. S. (2016). Mapa do Ensino Superior no Brasil. Recuperado de http://convergenciacom.net/pdf/mapa-ensinosuperior-brasil-2015.pdf

Silius, K.; Tervakari, A. (2003). The Usefulness of Web-based Learning Environments: The evaluation Tool into the Portal of Finnish Virtual University. International Conference on Network Universities and E-learning, Valencia.

Silva, V. G. da, Hilário, A. C. J. S., Souza, R. M. S. de, \& Gomes, M. J. (2017). Um Comparativo de Métodos de Usabilidade Pedagógica em Ambientes Virtuais de Aprendizagem. In Challenges 2017: Aprender nas Nuvens - Learning in the Clouds (p. 1865-1883). Braga. Recuperado de https://repositorium.sdum.uminho.pt/bitstream/1822/4 6035/1/Chalenges_2017_VS-MJG.pdf

Slater, R., Pearson, V. K., Warren, J. P., \& Forbes, T. (2015). Institutional change for improving accessibility in the design and delivery of distance learning - the role of faculty accessibility specialists at The Open University. Open Learning: The Journal of Open, Distance and e-Learning, 30(1), 6-20. http://doi.org/10.1080/02680513.2015.1013528

Tranfield, D., Denyer, D., \& Smart, P. (2002).

Developing an evidence-based approach to management knowledge using systematic review. Advanced Management Research Centre: Crafield School of Management, 44(1234), 28.

UNESCO. (1990). World declaration on education for all and framework for action to meet basic learning needs. Paris. Recuperado de http://unesdoc.unesco.org/images/0012/001275/12758 3e.pdf

W3C. (2016). World Wide Web Consortium. Recuperado 29 de junho de 2017, de http://www.w3.org

WCAG. (2008). Web Content Accessibility Guidelines (WCAG) 2.0. Recuperado 28 de setembro de 2016, de http://www.w3.org/TR/WCAG20/

\section{Agradecimentos}

Este trabalho foi realizado durante uma bolsa de estudos apoiada pelo Programa de Cooperação Internacional CAPES / COFECUB na Universidade do Minho. Financiado pela CAPES - Brasil.

Este trabalho é parcialmente financiado pelo CIEd Centro de Investigação em Educação, projetos UID/CED/1661/2013 e UID/CED/1661/2016, Instituto de Educação, Universidade do Minho, através de fundos nacionais da FCT/MCTES-PT. 